\title{
A Discrete Homotopic Deformable Model Dealing with Objects with Different Local Dimensions
}

\author{
Yann Cointepas ${ }^{1}$, Isabelle Bloch ${ }^{1}$, and Line Garnero ${ }^{2}$ \\ 1 ENST, Département TSI - CNRS URA 820 \\ 46 rue Barrault, 75634 Paris Cedex 13 FRANCE \\ email: yann.cointepas@enst.fr \\ ${ }^{2}$ LENA - CNRS URA 654 \\ 47 Bd de l'Hôpital, 75651 Paris Cedex 13 FRANCE
}

\begin{abstract}
In this paper we introduce a deformable model based on cellular complexes. This model allows the representation of objects with different local dimensions, and has good topological properties. We define homotopic deformation on this model and prove that a local criterion can be used to characterize simple elements of the model. This criterion is used to build an homotopic deformable model that can be used for image processing.
\end{abstract}

\section{Introduction}

In some applications it is necessary to be able to represent volumes and surfaces in the same model. For example, if one wants to represent the surface of the brain cortex with accuracy, it is necessary to be able to model 2-dimensional structures because the cortex folds make this surface complicated. The cortex surface can, on some aspects, looks like a crumpled thick sheet of paper where thin structures in the folds are 2-dimensional (figure 1). It is not thus reasonably possible to find an analytical model to represent it. For the same reasons, the use of a parametric model, although possible, would result in a structure which is complicated and difficult to use. Various approaches were proposed to define a surface in the discrete three-dimensional space $Z^{3}$, the graph-theoretical approaches 131112, the approaches based on voxel boundary 17] and the combinatorial approaches [2]5]. But, in order to have a model that can represent objects of different dimensions and that can also be used as a deformable model, it is necessary to introduce a new model.

We present, in section 2 a model based on cellular complexes that allows the representation of $0-, 1-, 2$ - and 3-dimensional structures in the same model. Then, in section 3 we define homotopic deformations for this model. Using the good topological properties of the cellular complexes, we adapt the notion of simple points of a binary digital picture to our model, and introduce the notion of simple elements that are elements that can be deleted from the model without 


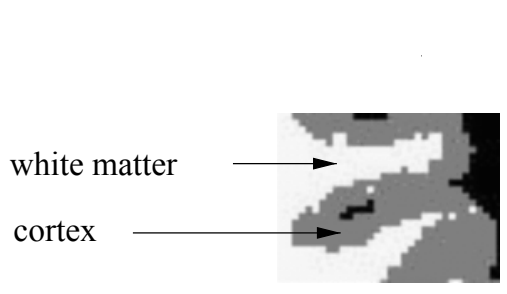

(a)

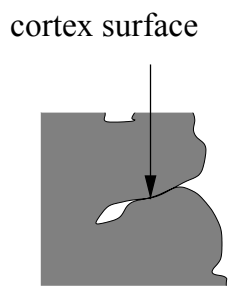

(b)

Fig. 1. Representation of thin structures: (a) Zoom on a slice of a segmented and classified brain image. (b) Brain volume and the cortex surface. The background is locally 2-dimensional in the folds.

changing its topology. We then prove that a unique local criterion can be used to characterize simple elements of any dimension. In the last section, we present a way to use this criterion to build an homotopic deformable model that can be used for the segmentation of medical images.

The classical surface definitions, when they are used with the conventional cubic grid, are not free from topological paradoxes. Moreover, while being based only on images made up of voxels, it is not possible to represent purely twodimensional structures. Indeed, either an element of surface is represented by a voxel in which case a surface is a three-dimensional structure, or a surface is made up of surfels, but in this case the membership of a surfel to the surface depends on the voxels in its neighborhood and it is therefore not possible to have purely two-dimensional objects because there is no surfel without voxel.

Therefore another approach has to be developed in order to model complex objects having parts of different local dimensions. Let us take the example of the brain cortex. In order to be able to model the cortex as well as possible, it is necessary to take into account the parts of the cortex surface that are inside the cortical folds. It is thus necessary to introduce a new modeling which makes it possible to represent at the same time volumes, to model the grey matter and the volumes of the cerebrospinal fluid (i.e. the background), and surfaces, to model the thin parts of the cortex surface. Moreover, in order not to have to re-sample the MRI, it would be interesting if this modeling remains close to the cubic grid. Lastly, this model must allow the study of the geometrical and topological properties of the objects it represents, if one wants to be able to use it as a deformable model. These constraints led us to develop a model based on cellular complexes.

\section{The cellular model}

The traditional digital images, in the sense that they use the cubic grid, are subject to several topological paradoxes [159] among which one of most famous is the connectivity paradox. To avoid this paradox, it is necessary to use two dis- 
tinct connectivity relations, one for the black components and one for the white components. But it does not lead to a consistent topological theory. Moreover, it is difficult, and in some case impossible, to transfer the most important topological notions such as that of an open subset, open neighborhood, continuity, topological mapping and many others.

On the basis of these observations and seeking a representation offering a consistent topological theory, Kovalevsky 10 emphasized the need for a structure made up of elements of various dimensions. Such a structure is known in topology as a cellular complex 6 14]. Thus, he introduced a representation of an image based on the elements (or cells) of different dimensions which compose the cubic grid. These elements are the cubes (or voxels), the faces (or surfels), the segments and the points. A mapping between the cells and a set of labels can be considered as an image and is called a cellular model. In this paper we only consider binary cellular models. The connectivity between the elements of a cellular model is based on a boundary relationship. Two elements are connected (or neighbors) if one bounds the other. For example, a cube is bounded by six faces, twelve segments and eight points. It has twenty six neighbors. A face is bounded by four lines and four points but it bounds two cubes so it has 10 neighbors (figure 2). There is only one connectivity relationship that does not depend on the labels of the cells but only on the dimension of the cells. Therefore, unlike classical digital pictures, the underlying graph is fixed, it does not depend on the image labels. Kovalevsky showed that this structure is free from the connectivity paradox.

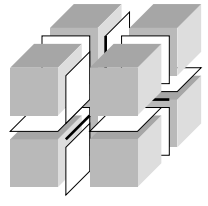

(a)

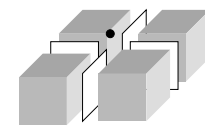

(b)

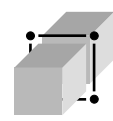

(c)

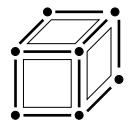

(d)

Fig. 2. The four neighborhoods of a cellular model: (a) the neighborhood of a point, (b) the neighborhood of a segment, (c) the neighborhood of a face and (d) the neighborhood of a cube.

This structure allows the representation of objects with different local dimensions. It is thus possible, for example, to have pure 2-dimensional structures and 3 -dimensional objects in the same model. The topology of the object that can be modeled with cellular model can be very complicated. If we want to be able to deform an object by deleting or adding elements, it is therefore necessary to preserve some topological properties of the object in order to be able to avoid creating complicated objects from simple ones. 


\section{Homotopic deformations}

Homotopic deformations are deformations that preserve certain topological properties. There is no unique definition of deformations that preserve topology in the literature. In the discrete three-dimensional topology framework, this notion is usually defined as the preservation of the number of connected components and of the number of tunnels [168]. This leads to the notion of simple points which are the voxels that can be removed from an object without changing its topology. Bertrand and Malandain [3] proposed a local characterization of simple points. In order to extend this concept to the cellular model, we will show that the same criterion can be used to characterize the simple elements of a binary cellular model.

At first, we will remain as general as possible and will define the concepts of path, loop and elementary deformation of a loop for abstract graphs. These notions allow the definition of simple elements of an abstract graph. Then, we will use these concepts within the framework of the cellular model to obtain a local characterization of the simple cells.

\subsection{Definitions}

Definition 1. An abstract graph $G$ is a pair $(E, V)$ where $E$ is a discrete set of abstract elements and $V$ is a set of ordered pairs $(x, y)$ where $x \in E$ and $y \in E$. $V$ is called neighborhood relationship.

In the following we consider a non oriented graph $G=(E, V)$, i.e. $(x, y) \in$ $V \Leftrightarrow(y, x) \in V$, such that $\forall x \in E,(x, x) \in V$. We note $O$ a subset of $E$ and $\bar{O}=E-O$ the complementary of $O$.

Definition 2. Two elements $x$ and $y$ of $O$ are neighbors on $O$ (denoted by $x \stackrel{O}{\rightleftharpoons} y$ ) if and only if $(x, y) \in V$. The set of neighbors of an element $s$ of $E$ (not necessary in $O$ ) belonging to $O$ is denoted by $N_{O}(s)$.

Definition 3. A path $\gamma$ of $O$ is either the empty path $\gamma_{\emptyset}$, or a series $s_{0}, s_{1}, \ldots, s_{n} \quad(n \in \mathbb{N})$ of elements of $O$ such that $s_{i} \stackrel{O}{\rightleftharpoons} s_{i+1} \forall i \in[0, n-1]$. $s_{0}\left(\right.$ resp. $\left.s_{n}\right)$ is the initial (resp. final) element of $\gamma$. The set of paths of $O$ is denoted by $\Gamma_{O}$.

Definition 4. Given an abstract graph $G=\left(E, f_{E}\right)$ and a subset $O$ of $E$, two elements $x$ and $y$ of $O$ belong to the same connected component of $O$ (denoted by $x \stackrel{O}{\longleftrightarrow} y$ ) if and only if there exists a path in $\Gamma_{O}$ of which $x$ is the initial element and $y$ the final element. The number of connected components of $O$ is denoted by $\mathrm{N}_{C C}(O)$.

Definition 5. The concatenation of two paths $\gamma=s_{0} . s_{1}, \ldots, s_{n}$ and $\gamma^{\prime}=s_{0}^{\prime}, s_{1}^{\prime}, \ldots, s_{m}^{\prime}$ of $\Gamma_{O}$, such that $s_{n} \stackrel{O}{\rightleftharpoons} s_{0}^{\prime}$, is the path $\gamma \cdot \gamma^{\prime}=s_{0}, s_{1}, \ldots, s_{n}, s_{0}^{\prime}, s_{1}^{\prime}, \ldots, s_{m}^{\prime}$. The empty path is the null element for concatenation: $\forall \gamma, \gamma_{\emptyset} \cdot \gamma=\gamma \cdot \gamma_{\emptyset}=\gamma$. 
Definition 6. A loop $\omega$ of $O$ is a series $s_{1}, s_{2}, \ldots, s_{n} n \in \mathbb{N}^{*}$ of elements of $O$, such that $s_{n} \stackrel{O}{\rightleftharpoons} s_{0}$ and $\forall i \in[1, n-1], s_{i} \stackrel{O}{\rightleftharpoons} s_{i+1}$. The set of loops of $O$ is denoted by $\Omega_{O}$.

In order to define topological equivalence we need to be able to characterize a tunnel. There is a tunnel in an object when one can find a loop that cannot be continuously deformed into a point in the object. Therefore we need to introduce a notion similar to the continuous deformation of a loop. We will use the notion of elementary deformation of a loop which is, intuitively, the smallest possible continuous deformation. The iteration of elementary deformations on a loop forms a set of deformations that are similar to the continuous deformations.

Definition 7. Two loops $\omega$ and $\omega^{\prime}$ of $O$, are equivalent up to an elementary deformation (denoted by $\omega \stackrel{O}{\rightleftharpoons} \omega^{\prime}$ ) if at least one of the following conditions is true:

$$
\begin{aligned}
& \text { - } \omega^{\prime} \stackrel{O}{\rightleftharpoons} \omega \\
& \text { - }\left\{\begin{array}{l}
\omega=s_{1} \cdot \pi \cdot s_{2} \\
\omega^{\prime}=s_{2} \cdot s_{1} \cdot \pi
\end{array} \text { where } \pi \in \Gamma_{O}, s_{1}, s_{2} \in O\right. \\
& \text { - }\left\{\begin{array}{l}
\omega=\pi_{1} \cdot s_{1} \cdot s_{2} \cdot s_{3} \cdot \pi_{2} \\
\omega^{\prime}=\pi_{1} \cdot s_{1} \cdot s_{3} \cdot \pi_{2}
\end{array} \text { where } \pi_{1}, \pi_{2} \in \Gamma_{O}, s_{1}, s_{2}, s_{3} \in O\right.
\end{aligned}
$$

The condition (1) imposes symmetry. The condition (2) allows two loops that differ only by a circular permutation on their elements to be equivalent. The condition (3) defines an elementary deformation as a "small deviation" of a loop on three elements which are mutually neighbors (figure 3). Since an abstract graph has no geometrical basis, it is important to define in what sense this relation can be seen as a continuous deformation. To choose a mapping between a graph and geometrical structure, one must consider the neighborhood relationship as a proximity relationship. Two elements are neighbors if they are "as close as possible". To compare the elementary deformation to a continuous deformation, it is sufficient that any geometrical structure composed of one element, two neighbors or a triplet of mutually-neighboring elements has the topology of a filled sphere. To deform a loop it is necessary to transform a pair of neighbors into a triplet of neighbors or to do the contrary. Then, any path in the object corresponding to a pair of neighbors $(x, y)$ can be continuously deformed into any other path in the object corresponding to a triplet of neighbors $(x, y, z)$. Therefore, the elementary deformation can be seen as a continuous deformation.

We are now able to continuously deform a loop by using a series of elementary deformations. This gives us the following equivalence relationship between loops:

Definition 8. Two loops $\omega$ and $\omega^{\prime}$ of $\Omega_{O}$ are equivalent by deformation if and only if at least one of the following conditions is verified:

- $\omega^{\prime} \stackrel{O}{\rightleftharpoons} \omega$

- there is a series $\omega_{0}, \omega_{1}, \ldots, \omega_{n}(n \in \mathbb{N})$ of loops of $O$ such that

$$
\omega \stackrel{O}{\rightleftharpoons} \omega_{0}, \omega_{0} \stackrel{O}{\rightleftharpoons} \omega_{1}, \ldots, \omega_{n-1} \stackrel{O}{\rightleftharpoons} \omega_{n}, \omega_{n} \stackrel{O}{\rightleftharpoons} \omega^{\prime}
$$




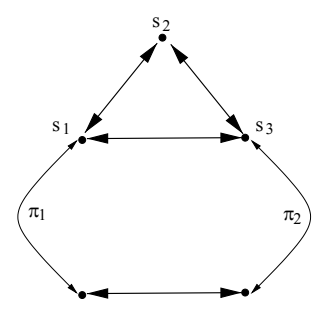

Fig. 3. Elementary deformation of a loop. The loop $\pi_{1} \cdot s_{1} \cdot s_{2} \cdot s_{3} \cdot \pi_{2}$ is equivalent to the loop $\pi_{1} \cdot s_{1} \cdot s_{3} \cdot \pi_{2}$.

The "equivalence by deformation" relationship is an equivalence relationship on $\Omega_{O}$. The transitivity is an immediate consequence of the definition. The symmetry is a consequence of the symmetry of $\omega \stackrel{O}{\rightleftharpoons} \omega^{\prime}$. The reflexivity is a consequence of the reflexivity of the neighborhood relationship. For any $\omega$ in $\Omega_{O}$, the equivalence class of loops of $O$ which contains $\omega$ is denoted by $[\omega]_{O}$.

In order to characterize tunnels we need to use the deformation of a loop into a point. Therefore, we will need the following definition.

Definition 9. A loop $\omega$ of $\Omega_{O}$ is reducible in $O$ if and only if there exists a loop $\omega^{\prime} \in[\omega]_{O}$ such that $\omega^{\prime}=s, s \in O$.

\subsection{Simple elements}

Kong and Rosenfeld [8] give the following definitions of an image and of simple points:

A conventional digital picture is a quadruple $(V, m, n, P)$, where $V=Z^{2}$ or $V=Z^{3}, P \subseteq V$, and where $(m, n)=(4,8)$ or $(8,4)$ if $V=Z^{2}$ and $(m, n)=$ $(6,26),(26,6),(6,18)$ or $(18,6)$ if $V=Z^{3}$. The elements of $V$ are called the points of the digital picture. The points in $P$ are called the black points of the picture; the points in $V-P$ are called the white points of the picture.

Let $\mathcal{P}_{1}=\left(Z^{3}, m, n, P\right)$ be a three-dimensional digital picture. Then the deletion of the points in a subset $D$ of $P$ preserves topology if and only if the resulting picture $\mathcal{P}_{2}=\left(Z^{3}, m, n, P-D\right)$ satisfies the following conditions:

each black component of $\mathcal{P}_{1}$ contains exactly one black component of $\mathcal{P}_{2},(6)$ each white component of $\mathcal{P}_{2}$ contains exactly one white component of $\mathcal{P}_{1}(7)$ each closed path in $P$ can be digitally deformed in $P$ to a closed path in (8) $P-D$

whenever one closed path in $P-D$ can be digitally deformed in $P$ to

another closed path in $P-D$, the first closed path can also be digitally

deformed in $P-D$ to the second closed path.

This definition of the simple points ensures the preservation of the number of connected components of black points (6) and of white points (7), it ensures 
also the preservation of the tunnels in the black components by prohibiting the disappearance of a closed curve (8) and the appearance of a closed curve in the white components (9). On the basis of these properties, we will define the concept of simple elements of a cellular model by adding constraints for preservation of the loops in the white components, and by expressing these constraints using the definitions 1 to 9

Let $G=\left(E, f_{E}\right)$ be an abstract graph, and $O$ a non empty subset of $E$. We note $\bar{O}=E-O$ the complementary of $O$. The set of simple elements of $O$, denoted by $S_{O}$ is a subset of $O$ such that $\forall s \in S_{O}$, the following conditions are true:

- $\exists x \in O-\{s\}$ such that $x \stackrel{O}{\longleftrightarrow} s$

- $\exists x \in \bar{O}$ such that $x \stackrel{\bar{O}+\{s\}}{\longleftrightarrow} s$

- $\forall x, y \in O-\{s\}, \quad x \stackrel{O}{\longleftrightarrow} y \Rightarrow x \stackrel{O-\{s\}}{\longleftrightarrow} y$

- $\forall x, y \in \bar{O}, \quad x \stackrel{\bar{O}}{\longleftrightarrow} y \Rightarrow x \stackrel{\bar{O}+\{s\}}{\longleftrightarrow} y$

- $\forall \omega \in \Omega_{O}, \quad \omega$ is irreducible in $O \Rightarrow$ $\exists \omega^{\prime} \in[\omega]_{O}$ such that $\omega^{\prime} \in \Omega_{O-\{s\}}$ et $\omega^{\prime}$ is irreducible in $O-\{s\}$

- $\forall \omega \in \Omega_{O-\{s\}}, \quad \omega$ is irreducible in $O-\{s\} \Rightarrow$ $\omega$ is irreducible in $O$

- $\forall \omega \in \Omega_{\bar{O}+\{s\}}, \quad \omega$ is irreducible in $\bar{O}+\{s\} \Rightarrow$ $\exists \omega^{\prime} \in[\omega]_{\bar{O}+\{s\}}$ such that $\omega^{\prime} \in \Omega_{\bar{O}}$ et $\omega^{\prime}$ is irreducible in $\bar{O}$

- $\forall \omega \in \Omega_{\bar{O}}, \quad \omega$ is irreducible in $\bar{O} \Rightarrow$ $\omega$ is irreducible in $\bar{O}+\{s\}$

The conditions (10) to (13) ensure the preservation of the connected components of $O-\{s\}$ and of $\bar{O}$ when $s$ is removed from $O$ and added to $\bar{O} ; 10$ prevents the disappearance of an isolated element of $O$; 111) prevents the appearance of an isolated element in $\bar{O}$, (12) prevents the separation of one connected component in $O$ and (13) prevents the fusion of two connected components of $\bar{O}$. The conditions (14) to (17) prevent the appearance or the disappearance of a tunnel in $O$ and in $\bar{O}$; (14) prevents the deletion of a tunnel in $O$, (15) prevents the appearance of a tunnel in $O$; (16) prevents the appearance of a tunnel in $\bar{O}$ and (17) prevents the disappearance of a tunnel on $\bar{O}$. We could define the expressions dual to (12) and (13) in order to prevent the separation of one connected component of $\bar{O}$, and the fusion of two connected components of $O$. But since we only consider the deletion of an element of $O$, these conditions would always have been true and thus are useless. Moreover, it is possible to add elements to $O$ while preserving the topology, we just have to swap $O$ and $\bar{O}$. This is why $O$ and $\bar{O}+\{s\}$ as well as $\bar{O}$ and $O-\{s\}$ have symmetrical roles. This symmetry will appear in the local characterization of the simple elements. 
We now introduce a condition that is necessary for an abstract graph element to be simple. We will then show that this condition is not sufficient in the general case but is sufficient when applied to a cellular model.

Proposition 1. If $s$ is a simple element of $O$, then $N_{C C}\left(N_{O}(s)-\{s\}\right)=$ $N_{C C}\left(N_{\bar{O}}(s)\right)=1$

Proof. Let $s$ be a simple element of $O$.

If $N_{C C}\left(N_{O}(s)-\{s\}\right)=0$, the condition (10) is not verified.

If $N_{C C}\left(N_{\bar{O}}(s)\right)=0$, the condition (11) is not verified.

If $N_{C C}\left(N_{O}(s)-\{s\}\right) \geq 2$ then there exists two distinct connected components $C_{1}$ et $C_{2}$ on $N_{O}(s)-\{s\}$. Therefore, there are two possibilities:

Either $C_{1}$ and $C_{2}$ are not connected in $O-\{s\}$, then $\exists x \in C_{1}$ and $\exists y \in C_{2}$ which do not verify (12), because $C_{1}$ and $C_{2}$ are connected in $O$ through $s$. Therefore $s$ is not a simple element of $O$.

Or, $\exists \gamma \in \Gamma_{O}, \exists s_{1} \in C_{1}, \exists s_{2} \in C_{2}$ such that $\omega=s_{1} . s . s_{2} \cdot \gamma \in \Omega_{O}$ (figure 4). For any number $n$ of deformations applied to $\omega$, we will alway obtain, except for a circular permutation, a loop $\omega_{n}$ such that:

$$
\omega_{n}=\pi_{1} . s_{1}^{\prime} . s . s_{2}^{\prime} \cdot \pi_{2} \text { where } s_{1}^{\prime} \in C_{1}, s_{2}^{\prime} \in C_{2} \text { and } \pi_{1}, \pi_{2} \in \Gamma_{O}
$$

Proof. Let us prove it by recurrence on $n$. If $n=0$, we have immediately $\omega_{0}$ by putting $s_{1}^{\prime}=s_{1}, s_{2}^{\prime}=s_{2}, \pi_{1}=\gamma_{\emptyset}, \pi_{2}=\gamma$. If the condition (18) is true for $n=N$ with $N \in \mathbb{N}$ then it is true for $n=N+1$ because an elementary deformation cannot delete $s$ from the loop, because $s_{1}^{\prime}$ and $s_{2}^{\prime}$ are not neighbors. Moreover the elementary deformation can only delete $s_{1}^{\prime}$ (resp. $s_{2}^{\prime}$ ) if there exists $s^{\prime \prime}$ in $C_{1}$ (resp. $C_{2}$ ) which would become the new predecessor (resp. successor) of $s$ in $\omega_{N+1}$ (figure[5). Therefore any loop of $[O]_{\omega}$ contains $s$ and at least two other elements, and therefore is irreducible and cannot belong to $\Omega_{O-\{s\}}$. Therefore $\omega$ is irreducible in $O$ and this contradicts (14). Therefore, $s$ is not a simple element of $O$.

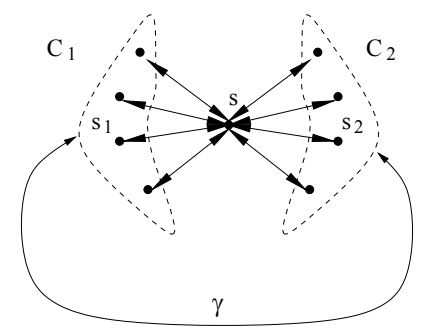

Fig. 4. In the neighborhood of $s$, there are two distinct connected components.

If $N_{C C}\left(N_{\bar{O}}(s)\right) \geq 2$ we can prove in the same way as before that (16) is not respected and therefore $s$ is not a simple element of $O$. 


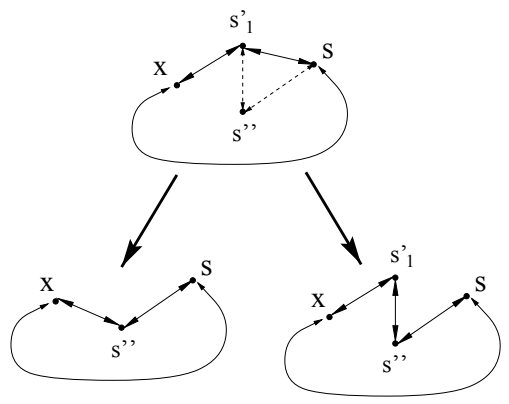

Fig. 5. Two possibilities of elementary deformation of a loop.

The reciprocal of the proposition 1 is not true. If $N_{C C}\left(N_{O}(s)-\{s\}\right)=$ $N_{C C}\left(N_{\bar{O}}(s)\right)=1$, the four connectivity conditions (10) to (13) are true. Moreover, let us show that(14) is true. If (14) were false, we would have:

$\exists \omega \in \Omega_{O}$ such that $\omega$ is irreducible in $O$ and such that

$\forall \omega^{\prime} \in \Omega_{O-\{s\}}$ such that $\omega^{\prime} \in[\omega]_{O}$ then $\omega^{\prime}$ is irreducible in $O-\{s\}$.

If $\omega^{\prime}$ exists, since it is equivalent to $\omega$ and irreducible in $O$, then $\omega$ is irreducible in $O$. It contradicts (19). Therefore $\omega^{\prime}$ does not exist, that means that all loops in $[\omega]_{O}$ contain $s$ and either $s$ is isolated $\left(N_{C C}\left(N_{O}(s)-\{s\}\right)=0\right)$, or there are at least two distinct connected components in $N_{O}(s)-\{s\}$. It contradicts the assumption that $N_{C C}\left(N_{O}(s)-\{s\}\right)=N_{C C}\left(N_{\bar{O}}(s)\right)=1$. Therefore (14) is true.

It is possible to prove in the same way that (16) is true too. But it is possible to find a set $E$, a neighborhood relationship $V$ and a subset $O \in E$ such that $N_{C C}\left(N_{O}(s)-\{s\}\right)=N_{C C}\left(N_{\bar{O}}(s)\right)=1$, and (15) is false or (17) is false (figure 6). This is because it is not possible to detect the appearance or disappearance of a tunnel with only the neighborhood of an element. To make it possible, it is necessary to have more assumptions about $E$ and $V$.

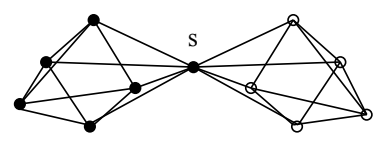

(a)

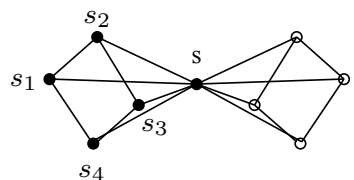

(b)

Fig. 6. (a) $s$ is a simple element of $O$. (b) $s$ is not a simple element of $O$ because the loop $s_{1} \cdot s_{2} \cdot s_{3} . s_{4}$ is reducible on $O$ but not on $O-\{s\}$. In both cases, $N_{C C}\left(N_{O}(s)-\{s\}\right)=N_{C C}\left(N_{\bar{O}}(s)\right)=1$. 


\subsection{Simple cells in a cellular model}

The neighborhood of an element of a cellular model depends on the type of element. There are four types of elements: cubes, faces, lines and points. But if we see the neighborhoods as a graph where the vertices are the elements of the complex and the arcs are the connectivity relationships, we can notice that there are only two different neighborhood graphs. Figure 7 shows that the different neighborhood types form only two distinct polyhedron shapes when we represent the connectivity graph. The polyhedron are made up of triangles which provide the following property:

$$
\forall s \in E, \forall s_{1}, s_{2}, s_{3} \in N_{E}(s), \quad s_{1} \stackrel{E}{\rightleftharpoons} s_{2} \text { and } s_{2} \stackrel{E}{\rightleftharpoons} s_{3} \Rightarrow s_{1} \stackrel{N_{E}\left(s_{2}\right)}{\longrightarrow} s_{3} .
$$
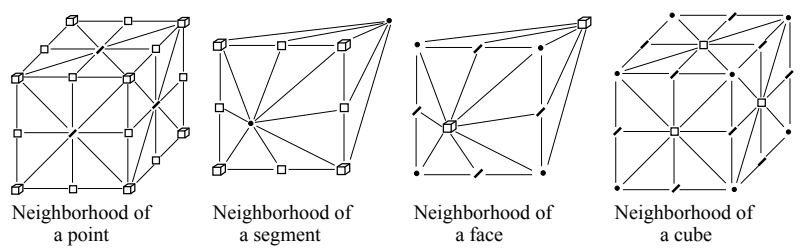

Fig. 7. The different neighborhoods of a cellular complex. Three-dimensional representation of the elements connected to a central element.

Proposition 2. If the neighborhood in $E$ of an element $s$ of $O$ can be represented as a polyhedron made up with triangles, then $N_{C C}\left(N_{O}(s)-\{s\}\right)=$ $N_{C C}\left(N_{\bar{O}}(s)\right)=1 \Leftrightarrow s \in S_{O}$.

Proof. We suppose $N_{C C}\left(N_{O}(s)-\{s\}\right)=N_{C C}\left(N_{\bar{O}}(s)\right)=1$. We have seen that we only have to show that the conditions (15) and (17) are true. We will prove it for the condition (15), the demonstration for (17) is similar. We will need the following lemma:

Lemma 1. If $s \in E$ is an element whose neighborhood in $E$ can be represented by a polyhedron made up with triangles and such that $N_{C C}\left(N_{O}(s)-\{s\}\right)=$ $N_{C C}\left(N_{\bar{O}}(s)\right)=1$, we have:

$\forall \omega \in \Omega_{N_{O-\{s\}}(s)}$, $\omega$ is reducible in $N_{O-\{s\}}(s)$

Proof. In order to avoid a tiresome proof we will use a geometrical approach that uses recurrence on the number $n$ of elements of $N_{\bar{O} s}()$.

If $n=1$, there exists only one element $s^{\prime}$ of $\bar{O}$ in $N_{E}(s)$. Since $N_{E}(s)$ can be represented as a polyhedron and since $s^{\prime}$ is a vertex of the polyhedron, the other vertices are elements of $O$ and can be represented as a planar structure made up with triangles. In this structure only the external vertices are connected to an 
element of $\bar{O}$ (figure 8 ). This structure represents the elements of $N_{O-\{s\}}(s)$ and is made up with triangles that represents triplets of elements, any pair of which being neighbors. Since an elementary deformation of a loop is based on such a triplet of elements, we can deduce that any loop of $N_{O-\{s\}}(s)$ is irreducible.

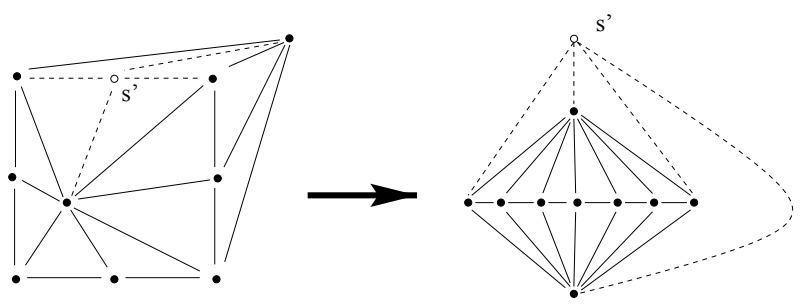

Fig. 8. Planar representation of a neighborhood.

Let us suppose that the lemma is true for $n=N$ where $N \geq 2$. To increment $n$ by one, we must remove an element from $N_{O-\{s\}}(s)$ to $N_{\bar{O}}(s)$. On the planar representation it is done by removing a point on the boundary of the structure because only the external points are connected to $N_{\bar{O}}(s)$. Thus, we do not change the properties of the planar structure and therefore any loop of $N_{O-\{s\}}(s)$ is reducible.

To prove the proposition 2, we will suppose that (15) is false:

$$
\exists \omega \in \Omega_{O-\{s\}} \text { such that } \omega \text { is irreducible in } O-\{s\} \text { and reducible in } O \text {. }
$$

Then:

$$
\omega \text { is reducible in } O \Rightarrow \exists \omega^{\prime} \in[\omega]_{O-\{s\}} \cap \Omega_{N_{O}(s)-\{s\}}
$$

According to the lemma, $\omega^{\prime}$ is reducible in $N_{O}(s)-\{s\}$, therefore $\omega$ is reducible in $O-\{s\}$, which contradicts (21).

\section{Cellular model implementation}

The best way to implement a cellular model is to use the structure of a classical image and replace each voxel with the cells presented in figure 9. Thus, we can use a four-dimensional array indexed by $(x, y, z, n)$ containing cells. The $n$ index is called the cell number and represents the cell type. It is possible to build the neighborhood system for each element using offsets on the array indices. Each cell of the figure 9 must be provided with its own set of offsets to find its neighbor, thus this set depends on the cell number $n$. Therefore, the cellular model implementation is composed of the following items :

- An array $C$ indexed by quadruplets $(x, y, z, n)$ and whose elements are cells. Indices $x, y$ and $z$ vary between zero and the cellular model size, $n$ is a cell number and varies between 0 and 7 . 
- Height neighborhood arrays $V_{0}, V_{2}, \ldots, V_{7}$ containing quadruplets $(d x, d y, d z$, $d n)$. For any element $(x, y, z, n)$ of $C$, each element of $V_{n}$ means that the element $(x+d x, y+d y, z+d z, n+d n)$ of $C$ is a neighbor of $(x, y, z, n)$.
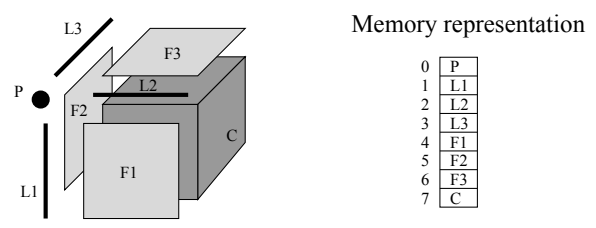

Fig. 9. Implementation of a cellular model.

It is clear that a cellular model is bigger than the corresponding image. Indeed, the implementation of a cellular model is about height times the size of a three-dimensional image. However, the structure we are using allows us to implement a cellular model in an efficient way since it is possible to represent a multi-dimensional array as a one-dimensional array and therefore to use an integer number $k$ to identify a cell. The cell number is then deduced from $k$ with a modulo operation. The neighborhoods are then four one-dimensional arrays of integers. As a result, algorithms using cellular models can be fast enough to deal with the large number of cells.

We can use the same method to pre-calculate in an array the connections between the neighbors of a cell which are necessary to decide if it is simple or not. It is therefore very fast to detect simple cells. Moreover, in the process of deforming a cellular model, we only consider the simple cells. If the shape of the objects modeled by the cellular model is not extremely complicated, the number of simple cells, which are on the border of the object, is low as compared to the number of cells. It is therefore possible to use this homotopic deformable model in a practical way.

\section{Discussion and Conclusion}

We showed that the only thing one has to know to decide if an element of a cellular complex is simple or not, is the neighborhood of the element. The local criterion used to characterize simple elements is easy to implement and fast to compute. Therefore, it is possible to use this criterion iteratively to deform an object without changing its topology. However, the order in which the simple elements are added or deleted is important because changing the label of an element can change the simpleness of its neighbors. Thus, it is difficult to parallelize such an algorithm and the result of a sequential algorithm can depend on the order used to scan the elements of the cellular complex. A good way to limit this order effect and to speed up the algorithm with parallelization, is to consider in a single pass only elements of the same dimension. Two elements of 
the same dimension are not neighbors, so the modification of one element does not influence the simpleness of the others. The result of such a single pass can therefore be independent of the scanning order if only local criteria are used to change the elements' labels.

In order to use this model as a deformable model, it is necessary to guide the model towards a desired solution. One possible way to do this is to use a cost function to choose the elements to modify. We successfully used this method to segment the human cortex from MR images of the head [4]. The global cost function we used was a combination of several cost functions that can be classified in two categories: external cost functions and internal cost functions. External cost functions use information that is not part of the model, for example localization information obtained after a classification of an image. Internal cost functions are obtained from the model and can be used to impose some geometrical a priori on the solution.

The modeling of an object with a cellular complex based model allows the representation of objects with different local dimensions, although such objects cannot be represented with conventional digital pictures. The good topological properties of cellular complexes allowed us to show that a cellular complex based object can be deformed while preserving its initial topology. The deformation is done by iteratively removing or adding simple elements from the object. We showed that a local criterion can be used to characterize the simple elements of an object. This simple criterion can be used to implement an homotopic multidimensional deformable model that can be used to solve image processing problems such as segmentation of objects with a complicated surface.

\section{References}

1. E. Artzy, G. Frieder, and G. T. Herman. The theory, design, implementation and evaluation of a three-dimensional surface detection algorithm. Computer Vision, Graphics and Image Processing: Graphical Models and Image Processing, 15:1-24, 1981.

2. Gilles Bertrand and Michel Couprie. Some structural properties of discrete surfaces. In Proceedings of DGCI'97, volume 1347 of LNCS, pages 113-124, 1997.

3. Gilles Bertrand and Grégoire Malandain. A new characterization of threedimensional simple points. Pattern Recognition Letters, 15:169-175, February 1994.

4. Yann Cointepas, Isabelle Bloch, and Line Garnero. Cellular complexes: A tool for $3 \mathrm{~d}$ homotopic segmentation in brain images. In Proceedings of ICIP'98, volume 3, pages 832-836, Chicago, 1998.

5. J. Françon. Discrete combinatorial surfaces. Computer Vision, Graphics and Image Processing: Graphical Models and Image Processing, 57:20-26, 1995.

6. Claude Godbillon. Eléments de topologie algébrique. Hermann, 1971.

7. Gabor T. Herman. Discrete multidimensional Jordan surfaces. Computer Vision, Graphics and Image Processing: Graphical Models and Image Processing, 54(6):507-515, 1992.

8. T. Y. Kong and A. Rozenfeld. Digital topology: Introduction and survey. Computer Vision, Graphics, and Image Processing, 48:357-393, 1989. 
9. V. A. Kovalevsky. Discrete topology and contour definition. Pattern Recognition Letters, 2(5):281-288, 1984.

10. V. A. Kovalevsky. Finite topology as applied to image analysis. Computer Vision, Graphics, and Image Processing, 46:141-146, 1989.

11. Grégoire Malandain, Gilles Bertrand, and Nicolas Ayache. Topological segmentation of discrete surfaces. International Journal of Computer Vision, 10(2):183-197, 1993.

12. Réy Malgouyres. A definition of surfaces of $Z^{3}$. In Conference on Discrete Geometry for Computer Imaging, pages 23-34, 1994.

13. D.G. Morgenthaler and A. Rosenfeld. Surfaces in three-dimensional images. Information and control, 51:227-247, 1981.

14. J. R. Munkers. Elements of Algebraic Topology. Addison-Wesley, Menlo Park. CA, 1984.

15. T. Pavlidis. Structural Pattern Recognition. Springer-Verlag, New York, 1977.

16. Y. F. Tsao and K. S. Fu. A parallel thinning algorithm for 3-d pictures. Computer Vision, Graphics, and Image Processing, 17:315-331, 1981. 